\title{
Partitioning Method in Noise Reduction to Improve Hand Detection
}

\author{
Bo Shen Woun, Guat Yew Tan, Ya Ping Won, and Edmund Ng
}

\begin{abstract}
This paper presents a multi-step algorithm for noise reduction in hand detection by using partitioning method. The main idea of the partitioning method is to divide an image into equal-size blocks, and obtain the percentage of number of pixels with skin color (skin pixels) in each block. Each of the blocks is labelled as either noise or part of the hand based on the percentage of skin pixels in that block. The image is then refined by repeating the processes to work on smaller blocks in the image with different comparison percentage values. The result of this paper shows partitioning method not only can filter the noise, but it also preserves the shape of the hand better, as compared with the Mathematical Morphology algorithm.
\end{abstract}

Index Terms-Hand detection, noise reduction, human computer interaction, partitioning method.

\section{INTRODUCTION}

Image noise is a major issue yet unavoidable in image processing. Noisy images are produced mainly during image sampling or transmission. Various types of noise such as photo electronic noise, impulse and structured noise present challenging problems in image restoration, particularly in object detection. Several noise reduction techniques have been developed to overcome different types of noise and for different aspects of image restoration.

Adaptive noise reduction algorithms such as median filtering, K-nearest neighbor averaging, gradient inverse weighted smoothing, etc. have been introduced in [1]-[3]. Median filtering is particularly effective for reducing impulse noise. It is performed by running through the entire image from one region to another, and replaces each pixel in question with the median of its neighboring entries. However the median based filter often cannot differentiate the thin lines and impulse noises and thus mistakenly removes the thin lines [4].

Fuzzy image filtering is a nonlinear processing technique used widely as an alternative to linear adaptive filtering. Mathematical morphology uses fuzzy image filtering to extract image components such as boundaries and skeletons;

Manuscript received December 19, 2012; revised February 21, 2013 This work was supported by the Malaysian Ministry of Higher Education under Exploratory Research Grant Scheme (ERGS) with reference number 203/PMATHS/6730018

Bo Shen Woun and Guat Yew Tan are with School of Mathematical Sciences, Universiti Sains Malaysia, 11800 Minden, Penang, Malaysia (e-mail: wbs102439@student.usm.my,gytan@cs.usm.my).

Ya Ping Wong is with Faculty of Computing and Informatics, Multimedia University, 63100 Cyberjaya, Malaysia (e-mail: ypwong@mmu.edu.my).

Edmund $\mathrm{Ng}$ is with Faculty of Cognitive Sciences and Human Development, Universtiy Malaysia Sarawak, 94300 Kota Samarahan, Sarawak, Malaysia (e-mail: gwng@fcs.unimas.my). and also to filter the image noise using erosion processes [5], [6]. However, by using morphology in hand detection, the end effect tends to distort the size of the hand.

In our research, we propose to use partitioning method in image noise reduction to improve hand detection for an image with background color close to skin color. Our proposed methodology divides an image equally into blocks. The percentage of number of pixels with skin color (skin pixels) in each block is calculated and compared against a threshold value (skin threshold) to decide if the block is noise or part of the hand. A comparison between partitioning method and morphology algorithm is made, particularly on image noise reduction in hand detection. The result produced by partitioning method displays a cleaner hand image with better shape preservation.

\section{Methodology}

Our project uses partitioning method, which adopts divide-and-conquer strategy to reduce the noise of an image. The whole methodology is divided into 5 steps, including a pre-processing step. In this paper, we apply our noise reduction algorithms to improve the result of hand detection.

\section{A. Step 1 (pre-process): Background Subtraction}

A moving hand can be recognized from a set of still images by background subtraction algorithms accurately and efficiently [7], [8]. However, if the image background contains a color close to the target hand skin color, the resulting hand obtained may not be satisfactory. This is due to the colors in some parts of the hand may coincidentally match the background color and considered by the algorithm as background. Referring to Fig. 1 and Fig. 2, we consider 2 images, $I N_{1}$ (Fig. 1) and $I N_{2}$ (Fig. 2), for background subtraction discussion. Initially, both source images are converted to gray scale images for more accurate comparison. Traditional background subtraction algorithm is used. In Fig. $3, H A N D_{1}$ is produced by using the following equation(1) [8].

$$
\operatorname{HAND}_{1}(x, y)=\left\{\begin{array}{c}
255, \text { if }\left|I N_{2}(x, y)-I N_{1}(x, y)\right| \geq T_{1} \\
0, \text { otherwise }
\end{array}\right.
$$

where $H A N D_{1}(x, y), I N_{1}(x, y)$ and $I N_{2}(x, y)$ are brightness of the pixels at coordinates $(x, y)$ for images $H A N D_{1}, I N_{1}$ and $I N_{2}$ respectively. $T_{1}$ is the threshold value set to adjust the sensitivity of detecting the foreground object.

The result of background subtraction is displayed in Fig. 3, where the hand and arm have been set to white, denoting the foreground object. The curtain has also been set to white, because the curtain was not totally stationary but moved 
slightly by the breeze.

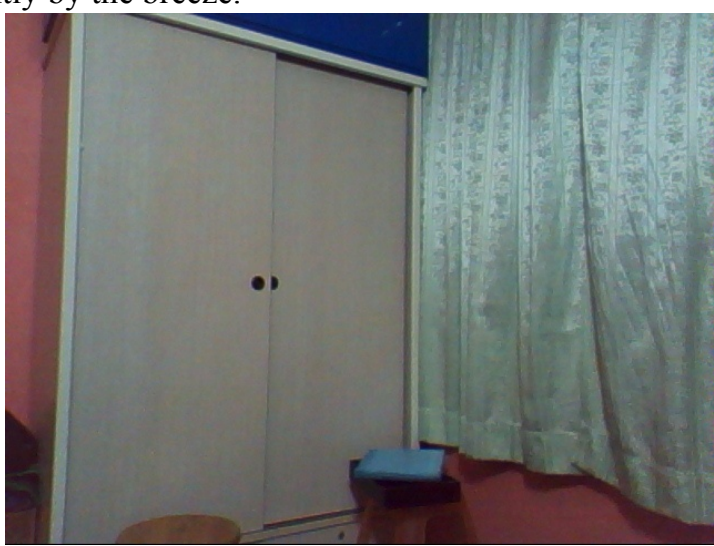

Fig. 1. Image $I N_{1}$, which is the background of Image $I N_{2}$

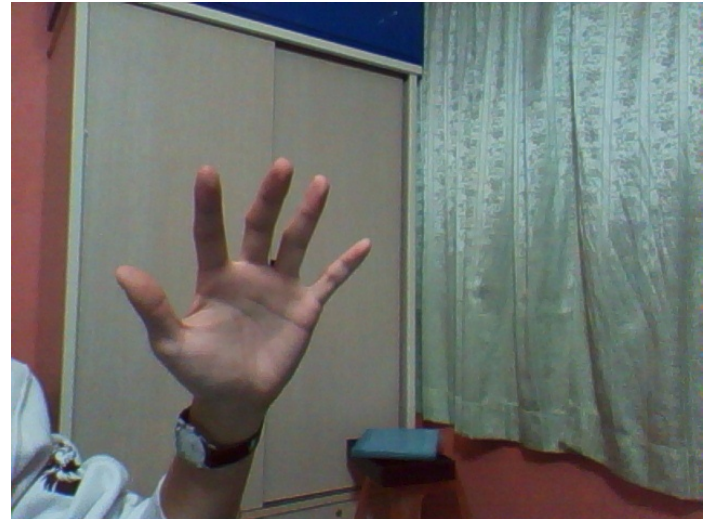

Fig. 2. Image $I N_{2}$. Note that the door's color is close to the skin color of the hand.

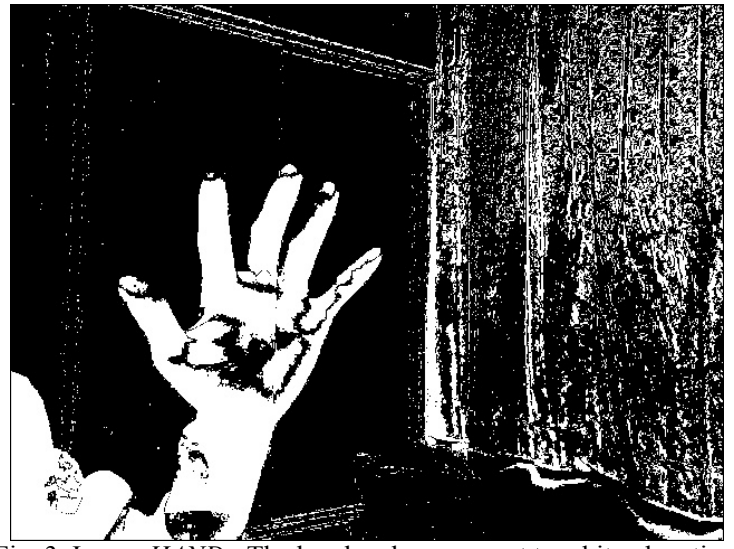

Fig. 3. Image $H A N D_{1}$. The hand and arm are set to white, denoting foreground. Curtains are also set to white as it is moving due to the breeze.

\section{B. Step 2: Skin Color Detection - Pass 1}

Step 2 eliminates any foreground object which is non-hand by comparing the color of the foreground object with skin color. The pixels coordinates for the foreground objects are obtainable from Image $H A N D_{1}$. By using these coordinates, we refer to Image $I N_{2}$ and convert the RGB colors of the corresponding pixels in Image $I N_{2}$ to HSV colors. After the conversion, we compare hue value of the corresponding pixels in Image $I N_{2}$ within the range of $0-60$ and 300-360, where researches have shown that the hue values of human skin color are more prone to fall under these ranges [9]-[11]. For pixels fall under these ranges, white color is assigned to denote the hand and everything else to black. In Fig. 4, note that the sleeve and wrist watch pixels in Image $H A N D_{2}$ image are assigned to black. Most of the curtain pixels are also set to black.

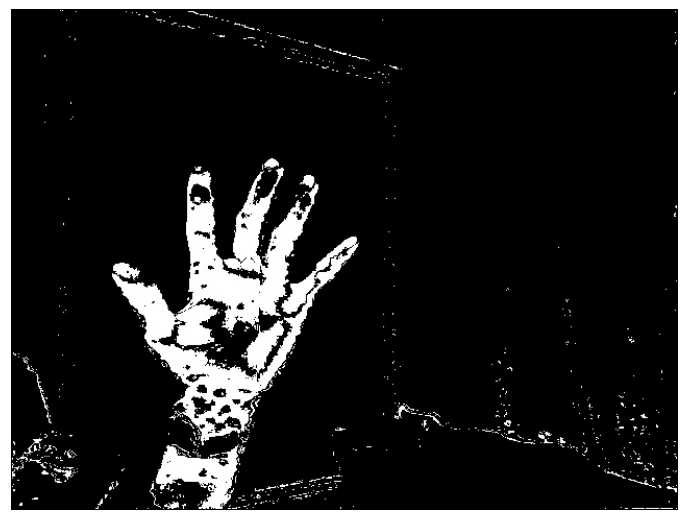

Fig. 4. Image $\mathrm{HAND}_{2}$. The curtain, sleeve and wrist watch pixels are set to black, to denote the non-hand pixels.

\section{Step 3: Partitioning the image-Pass 1}

It is common that noises are yielded between two consecutive images frames in a video stream. However, background subtraction algorithm will consider the noise as the difference between two images and thus capture the pixels as a foreground object. Refer to Fig. 3, pixels of the static door frame is set to white after background subtraction, denoting presence of noises, and the hue value of the noises fall under the skin color hue range coincidentally (Fig. 4). Though the areas of the noisy objects captured are much smaller as compared to the area of a hand, we try to achieve a clean image which contains only hand but nothing else. Further, note that the hand contains "holes" (black pixels) especially at the palm center and at the fingers. This is because the colors at those parts are close to the color of their corresponding background. Step 3 tries to solve these 2 problems.

In this step, Image $H A N D_{2}$ is divided into blocks with sizes $50 \times 50$ pixels each. Going through block-by-block, the percentage of white pixels in each block are counted. White pixels in Image $H A N D_{2}$ denote either noise or hand. If there are less than $85 \%$ of white pixels present in the block, we assume the white pixels are noise and the whole block is set to black. We define the value of 85 as skin threshold. On the contrary, if the percentage of white pixel is greater than skin threshold, the block is assumed to be part of the hand and set to white. The result is shown in Fig. 5, the noise in $H A N D_{2}$ is filtered in Image $\mathrm{HAND}_{3}$.

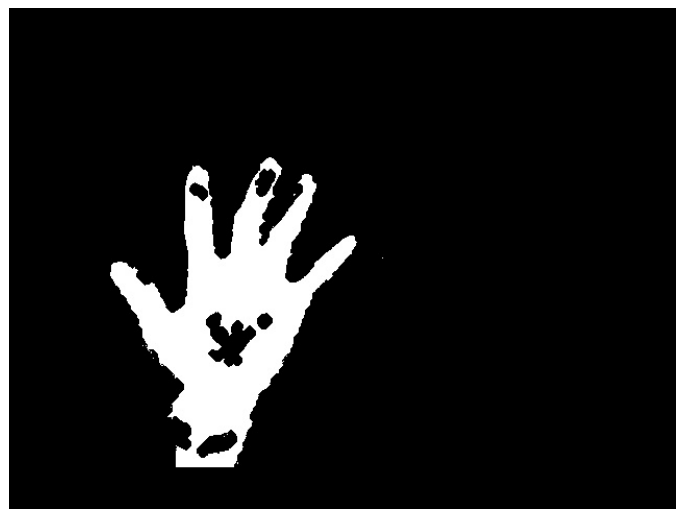

Fig. 5. Image $\mathrm{HAND}_{3}$. Result of Step 3 in Pass 1 partitioning.

\section{Step 4: Skin Color Detection-Pass 2}

Step 3 (Image $\mathrm{HAND}_{3}$ ) has successfully eliminated the noise in Image $H_{A N D_{2}}$. However, the shape of the hand is unsatisfactory. Though the hand looks much better than 
$H A N D_{2}, H A N D_{3}$ still contains holes especially at the fingers and palm center. In this step, we run a skin color detection for the second time but with some variations from the detection process in step 2. Let $p$ be the pixel coordinate for the hand (white pixels) in Image $H A N D_{3}$. The hue values of neighboring pixels for $p$ in the HSV version of Image $I N_{2}$ in step 2 is compared against a wider range of hue values, 0-100 and 270-360. The wider range of the hue values is to accommodate the color in some parts of the hand, which is close to the background color. Wider range is set in this step as the neighboring pixels of $p$ under consideration have higher chance to be part of the hand. The resulting image is shown in Fig 6. The holes at the fingers are fill and palm center looks more solid, however, some of the pixels are incorrectly assigned to white.

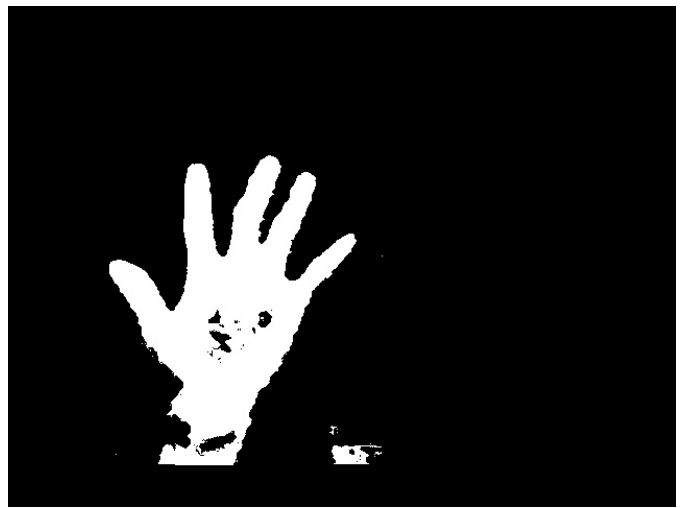

Fig. 6. Image $\mathrm{HAND}_{4}$ image. The fingers holes are filled and hollow areas are more solid at the palm center. However, note that some of the pixels are incorrectly assigned to white.

\section{E. Step 5: Partitioning the image-Pass 2}

This step further improves the image quality of Image $H A N D_{4}$. The small white patches in Image $H A N D_{4}$ (Fig. 6) can be considered as noise and removed by partitioning the image as in step 3 with some variations. Image $\mathrm{HAND}_{4}$ is divided equally into smaller blocks with sizes $10 \times 10$ pixels each. Similar to Pass 1, the percentage of number of white pixels is counted and upon reaching the skin threshold of $85 \%$, the block is set to white. The result is displayed in Fig. 7. Image $H_{A N D}$ contains the hand images which shows better result compare to morphology algorithm in Fig. 8.

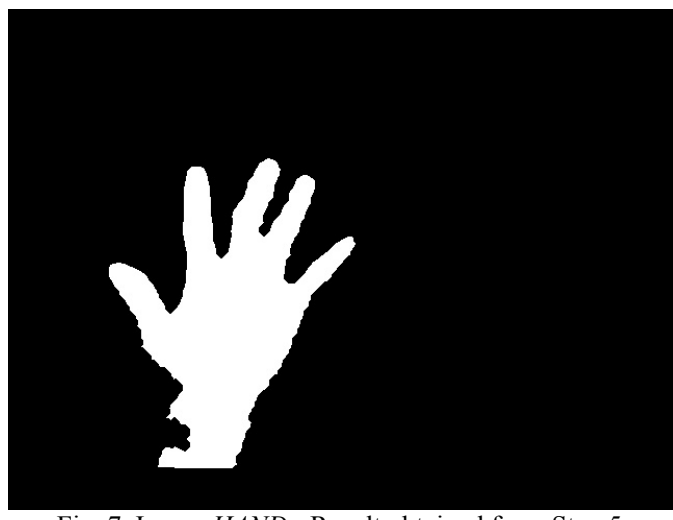

Fig. 7. Image $H A N D_{5}$. Result obtained from Step 5

We have used Mathematical Morphology algorithm to repair Image $H A N D_{1}$ and the result is shown in Fig 8. Compare to Image $H A N D_{5}$, morphology does not solve the hollow palm centre problem efficiently and the size of the hand is enlarged noticeably. Further, noises are obviously presence as white dots in the image.

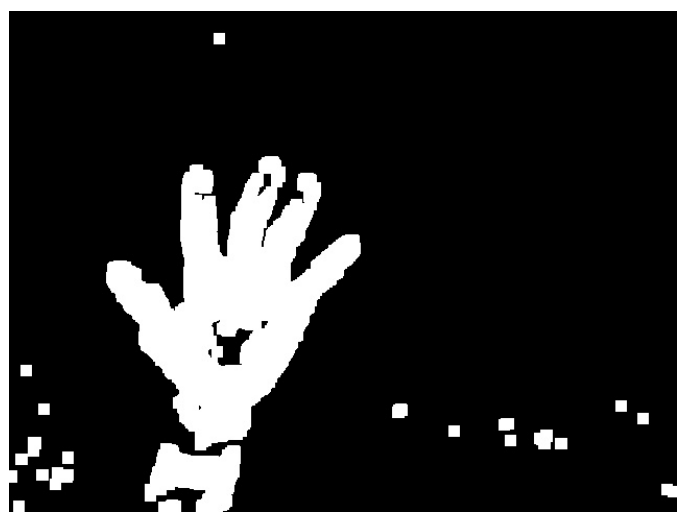

Fig. 8. Image MORPH, produced by applying Morphology algorithm to Image $H A N D_{1}$

\section{ALGORITHM FRAMEWORK}

This section displays the algorithm framework which was discussed in detail in the previous section.

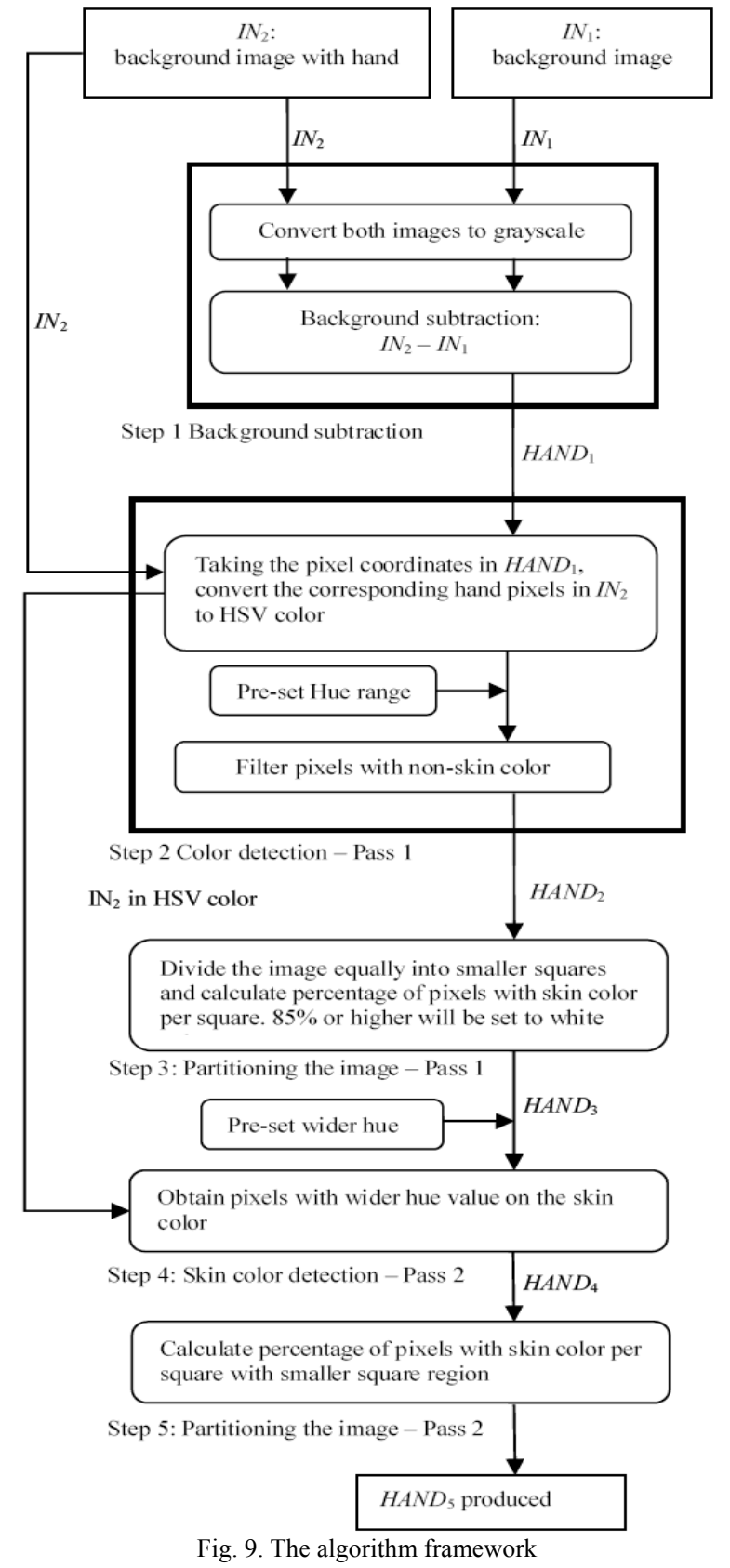

Fig. 9. The algorithm framework 


\section{FUtURe Plan}

This project serves as the first phase in finger detection in a $3 \mathrm{D}$ environment. The future plan includes convex hull detection, contour line sketching, etc.

\section{REFERENCES}

[1] G. A. Mastin, "Adaptive filters for digital image noise smoothing: an evaluation," Comp. Vision, Graph., Image Process., vol. 31, pp. 103-120, 1985.

[2] J. Rasure, D. Argiro, T. Sauer, and C. Williams, "A visual language and software development environment for image processing," Int. J. Imag. Syst. and Technol., vol. 2, pp. 183-199, 1990.

[3] J. S. Weszka and A. Rosenfeld, "Threshold evaluation techniques," IEEE Trans. Syst, Man, and Cybern., vol. SMC-8, no. 8, pp. 622-629, 1978.

[4] S. Zoican, "Improved median filter for impulse noise removal," TELSIKS Serbia and Motenegra, Nis, vol. 10, no. 123, pp. 681-684, 2003.

[5] C. R. Giardina and E. R. Dougherty, Morphological Methods in Image and Signal Processing, New Jersey: Prentice-Hall, Englewood Cliffs, 1988.

[6] R. M. Haralick, S. R. Sternberg, and X. Zhuang, "Image analysis using mathematical morphology," IEEE Trans. Pattern Anal. Machine Intell. vol. PAMI-9, no. 4, pp. 532-550, 1987.

[7] S. Sakaida, M.Naemura, and Y. Kanatugu, "Moving object extration using background difference and region growing with spatio-temporal watersheds," Systems and Computers in Japan, vol. 33, no. 12, pp. 11-26, 15 November 2002.

[8] A. Ogihara, H. Katsumoto, and A. Shiozaki, "Hand region extraction by background subtraction with renewable background for hand gesture recognition," International Symposium on Intelligent Signal Processing and Communication Systems (ISPACS2006), Tottori, Japan.

[9] Chasan's Place-Skin Color Detection with HSV Lookup. [Online]. Available:

http://www.chasanc.com/index.php/Image-Processing/Skin-Color-Det Jamie ection-with-HSV-Lookup.html

[10] J. Sherrah and S. Gong. Skin Colour Analysis. [Online]. Available: http://homepages.inf.ed.ac.uk/rbf/CVonline/LOCAL_COPIES/GONG $1 /$

[11] M. Störring, H. Andersen, and E. Granum, "Estimation of the illuminant color from human skin color," in Proc. IEEE International Conference on Face \& Gesture Recognition, pp. 64-69, March 2000.

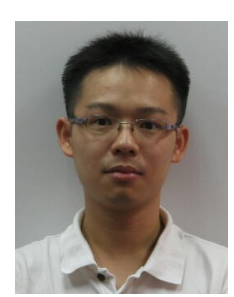

Bo Shen Woun graduated with Bachelor of Applied Science major in computer modeling in 2011, from Universiti Sains Malaysia (USM), Penang, Malaysia $\mathrm{He}$ is a full-time research student at USM at present

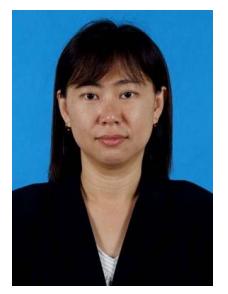

Guat Yew Tan graduated with Master of Applied Science (1996) from Nanyang Technological University, Singapore.

She is a senior lecturer at School of Mathematical Sciences, Universiti Sains Malaysia (USM), Malaysia Prior to her assignment in USM, she worked at the industrial sectors including consulting work in the area of Information technology.

Ms Tan is a life member in PERSAMA, Malaysia. She has written the book $C++$ Programming: An Introduction.

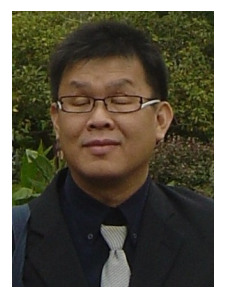

Ya Ping Wong is a principal lecturer at Faculty of Computing and Informatics, and researcher in Vision Lab at Multimedia University, Malaysia. His research interest is computer graphics, computer vision and machine learning.

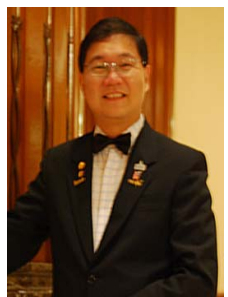

Edmund $\mathbf{N g}$ graduated with a $\mathrm{PhD}$ in advanced interactive and behaviroural technology (2007) from University of Salford, United Kingdom.

$\mathrm{He}$ is a senior lecturer at the Faculty of Cognitive Sciences and Human Development, University Malaysia Sarawak (UNIMAS) and the Director of Centre of Excellence for Semantic Technology and Augmented Reality in UNIMAS. His expertise is in the area of augmented reality, virtual reality and intelligent interactive system. His research interest is in the usability engineering, such as, human computer interaction, human factors in information systems, and the use of visual science and artificial intelligence to enhance trainer capability. 